\title{
Universality of many-body states in rotating Bose and Fermi systems
}

\author{
M. Borgh, ${ }_{1}^{1}$ M. Koskinen, ${ }^{2}$ J. Christensson, ${ }^{1}$ M. Manninen, ${ }^{2}$ and S. M. Reimann ${ }^{1}$ \\ ${ }^{1}$ Mathematical Physics, LTH, Lund University, 22100 Lund, Sweden \\ ${ }^{2}$ NanoScience Center, 40014 University of Jyväskylä, Finland
}

(Received 19 December 2007; published 14 March 2008)

\begin{abstract}
We propose a universal transformation from a many-boson state to a corresponding many-fermion state in the lowest-Landau-level approximation of rotating many-body systems, inspired by the Laughlin wave function and by the Jain composite-fermion construction. We employ the exact-diagonalization technique for finding the many-body states. The overlap between the transformed boson ground state and the true fermion ground state is calculated in order to measure the quality of the transformation. For very small and high angular momenta, the overlap is typically above $90 \%$. For intermediate angular momenta, mixing between states complicates the picture and leads to small ground-state overlaps at some angular momenta.
\end{abstract}

DOI: 10.1103/PhysRevA.77.033615

PACS number(s): 03.75.Mn, 03.75.Kk, 71.10.Pm, 68.65.-k

\section{INTRODUCTION}

The properties of rotating many-body systems have been the topic of intense study, theoretical as well as experimental [1-22]. (See also Ref. [23] for a very recent review.) In particular, Bose-Einstein condensates have been of great interest since the advent of the laser-cooling technique [24]. It is by now a well-established fact that quantum many-body systems under rotation form quantized vortices, a property that has been known in the contexts of superconductivity and superfluidity $[25,26]$. In earlier works, the formation of vortices in few-body systems was studied quite extensively, including both boson and fermion systems [10,17,27], as well as their two-component generalizations [27-29]. In Ref. [10] we noted that some properties of vortex formation in fewbody systems of repulsively interacting particles in the lowest Landau level are universal, not only with respect to the details of the interaction (the boson system was studied both with the long-range Coulomb interaction and with a shortrange, contact interaction), but also with respect to the statistics of the constituent particles. We found remarkable similarities between the yrast spectra of the boson and fermion systems, and we also noted that vortices enter at very specific, and corresponding, angular momenta in the boson and fermion systems, respectively.

In the present paper, we formalize this boson-fermion universality by means of a transformation from a bosonic manybody wave function to a corresponding fermionic wave function. The transformation is inspired by the Laughlin wave function [30], and forms a direct parallel to the ChernSimons transformation in the theory of the fractional quantum Hall effect $[31,32]$ and Jain's composite-fermion picture [33]. (The latter was recently successfully applied to the problem of small quantum dots in magnetic fields, and other small quantum systems at high angular momentum $[11-15,18,20,21]$.) This allows us to investigate quantitatively to what extent this universality holds in a general comparison between few-body boson and fermion systems in the lowest Landau level. The particles may be electrons in a quantum dot (in the case of fermions) or optically or magnetically trapped ions at ultralow temperatures. In this work we concentrate on harmonically confined, Coulomb- interacting particles in two dimensions. One might consider other model interactions. However, in a Fermi system, a contact or short-range interaction would result in huge (quasi)degeneracies, rendering the wanted overlaps essentially meaningless, as well as difficult to calculate. The Coulomb interaction provides a clear-cut test case for the proposed model. We will demonstrate in Sec. IV that our results seem to be robust with respect to changes in the details of the interaction. We therefore expect that the results of this paper would apply also, for example, to the realistic dipole interaction considered in Ref. [34].

This paper is organized as follows: The next section gives an introduction to the mathematics of the proposed bosonfermion universality of many-body systems in the lowest Landau level. This section also introduces the concepts used throughout the paper, and defines a mathematical transformation of a many-boson wave function into a corresponding many-fermion wave function. Section III gives a brief description of the exact-diagonalization method in the lowest Landau level, and of the implementation of the bosonfermion transformation. The reader who is familiar with exact diagonalization may skip directly to Sec. IV, where our results are presented and discussed. Some concluding remarks are given in Sec. V.

\section{INTRODUCTION TO BOSON-FERMION UNIVERSALITY}

In order to formulate a mathematical expression for the direct comparison between boson and fermion many-body wave functions, we use complex coordinates for the twodimensional plane, $z=x+i y$. The general wave function in the lowest Landau level of one particle in a harmonicoscillator potential is $\psi_{\ell}(z) \propto z^{\ell} e^{-|z|^{2}}$, where $\ell$ is the angular momentum. (Atomic units and one fixed frequency $\omega=1$ of the harmonic confinement are used throughout the paper.) In the boson system at zero angular momentum, all particles reside in the $\ell=0$ state, and the many-body wave function is

$$
\Psi_{0}^{B} \propto e^{-\Sigma_{k}\left|z_{k}\right|^{2}} .
$$

As the system is set rotating, particles are lifted from the $\ell$ $=0$ single-particle state in order to carry angular momentum. 
A single vortex is formed at the center of mass when the total angular momentum of the system equals the number of particles, in which case all particles are in the $\ell=1$ state. As angular momentum increases further, more vortices successively enter the system. As detailed in Ref. [10], a vortexgenerating state carrying $n$ vortices can be obtained from Eq. (1) by successive multiplications with symmetric polynomials:

$$
\begin{aligned}
\Psi_{n} & =\prod_{j_{1}}^{N}\left(z_{j_{1}}-a e^{i \alpha_{1}}\right) \times \cdots \times \prod_{j_{n}}^{N}\left(z_{j_{n}}-a e^{i \alpha_{n}}\right) \Psi_{0}^{B} \\
& =\prod_{j}^{N}\left(z_{j}^{n}-a^{n}\right) \Psi_{0}^{B} .
\end{aligned}
$$

This wave function describes a state with $n$ vortices evenly spaced on a ring with radius $a$ centered at the origin, and may be used to obtain a trial many-body wave function [10].

The same line of reasoning can be repeated completely analogously for fermions. However, in the lowest Landau level the angular momentum of a many-fermion system cannot be zero. Instead the smallest possible total angular momentum is achieved by putting one fermion in each of the $N$ lowest single-particle states with single-particle angular momenta ranging from 0 to $N-1$. This state is called the maximum density droplet (MDD) and is the fermion equivalent of the zero-angular-momentum states for bosons. The wave function of the MDD is given by the Laughlin wave function with the filling factor one [35]:

$$
\Psi_{\mathrm{MDD}}^{F} \propto \prod_{i<j}^{N}\left(z_{i}-z_{j}\right) e^{-\Sigma_{k}\left|z_{k}\right|^{2}} .
$$

In the construction of the vortex-generating state for the fermion system, the boson condensate is replaced by the MDD.

Now we compare Eq. (1) with Eq. (3) and note that the latter can be obtained from the former simply by multiplication with the polynomial

$$
D^{F}=\prod_{i<j}^{N}\left(z_{i}-z_{j}\right) .
$$

Making the assumption that the transformation between the lowest angular momentum states, $\Psi_{\mathrm{MDD}}^{F}=D^{F} \Psi_{0}^{B}$, holds whenever $\Psi_{0}^{B}$ is replaced by $\Psi_{\mathrm{MDD}}^{F}$, we arrive at a very general transformation from any bosonic many-body wave function to a corresponding fermionic wave function, shifted in angular momentum by exactly $L_{\mathrm{MDD}}$ :

$$
\Psi_{L_{\mathrm{MDD}}+L_{B}}^{F}=D^{F} \Psi_{L_{B}}^{B} .
$$

The degree of universality of the structure of the manybody wave function between boson and fermion systems will be reflected in how well the transformation (5) reproduces the true fermion wave function. The straightforward way of determining this is to calculate the wave function of a given many-fermion system both directly, in order to obtain the true wave function, and by transforming the corresponding boson state according to Eq. (5). The overlap between the true wave function and the transformed boson wave function can then be calculated:

$$
O=\left|\left\langle\Psi_{L_{\mathrm{MDD}}+L_{B}}^{F} \mid D^{F} \Psi_{L_{B}}^{B}\right\rangle\right|^{2} .
$$

The argument given above for the boson-fermion correspondence is entirely heuristic: it is based on observing the common features of fermion and boson wave functions in the lowest Landau level. The correspondence formalized in Eq. (5) is ultimately justified by the explicit demonstration of its performance in Sec. IV. However, it is reasonable to anticipate the existence of a boson-fermion transformation of this type from boson-Chern-Simons theory [31,32] and composite-fermion theory $[32,33]$. A fermionization of repulsively interacting bosons in the lowest Landau level has been described by several authors $[9,13-15,18,19,36]$. In particular, Cooper and Wilkin [36], Cazalilla et al. [14], Chang et al. [13], as well as Regnault et al. [18] explicitly map interacting bosons onto noninteracting, spinless fermions by means of a composite-fermion construction. It is known that the mean-field approximation of noninteracting composite fermions loses accuracy as angular momentum increases, and effects of residual interactions become important $[11,13,18,20]$. In the case of a harmonic interaction, the transformation (5) between interacting bosons and interacting fermions was rigorously derived recently by Ruuska and Manninen [37].

In the Chern-Simons approach to the fractional quantum Hall effect, the many-body wave function $\Psi_{e}$ of the electrons of the two-dimensional electron liquid is transformed into a wave function $\Psi_{\mathrm{CS}}$ of particles moving in an effective magnetic field. The transformation is defined as follows [32]:

$$
\Psi_{e}=\prod_{i<j}\left[\frac{z_{i}-z_{j}}{\left|z_{i}-z_{j}\right|}\right]^{p} \Psi_{\mathrm{CS}} .
$$

The prefactor determines the number of flux quanta associated with each particle, and it is symmetric or antisymmetric with respect to particle interchange depending on whether $p$ is chosen even or odd. In the simplest possible model, we choose $p=1$. Since the original electron wave function is fermionic, this means that the Chern-Simons wave function describes a corresponding boson system. The transformation between the boson system and the fermion system is given by the factor $\Pi_{i<j}\left[\left(z_{i}-z_{j}\right) /\left|z_{i}-z_{j}\right|\right]$.

Jain took the Chern-Simons approach further to a more sophisticated ansatz in the lowest Landau level by introducing his composite-fermion theory $[32,33]$. In this approach, the power $p$ of the Chern-Simons factor is explicitly taken to be an even integer, thereby making the composite particles fermions. Also, the prefactor is simply taken to be the $J a$ strow factor $\prod_{i<j}\left(z_{i}-z_{j}\right)^{p}$, which attaches vortices rather than flux tubes to the particles [32]. The wave function of the original fermions is retained by projection to the lowest Landau level: 


$$
\Psi_{e}=\mathcal{P} \prod_{i<j}\left(z_{i}-z_{j}\right)^{p} \Psi_{\mathrm{CF}}
$$

where $\mathcal{P}$ is the projection operator into the lowest Landau level.

Jain's construction and the bosonic Chern-Simons wave function immediately suggest a mapping between a boson system and an equivalent fermion system at some other magnetic field (or, equivalently, angular momentum) of the form described by Eq. (5). For a harmonic particle-particle interaction, this relation is exact, and can be derived analytically by constructing a spectrum-generating algebra, and applying the corresponding ladder operators to the ground-state wave functions $\Psi_{B}=1$ and $\Psi_{F}=\Pi_{i<j}\left(z_{i}-z_{j}\right)$, respectively [37].

The numerical study presented in this paper shows that the transformation is very general and yields a good approximation for the fermion wave function in the lowest Landau level, also for Coulomb-interacting particles.

\section{THE EXACT-DIAGONALIZATION METHOD AND THE BOSON-FERMION TRANSFORMATION}

In order to calculate the overlap, Eq. (6), the many-body wave functions must be obtained with sufficient accuracy. In this study we take as our model system harmonically confined, Coulomb-interacting particles, and we solve both the fermion and the boson problems with the exactdiagonalization method. The boson wave function is transformed into a corresponding fermionic many-body wave function by means of Eq. (5), which we have implemented numerically to operate on a bosonic wave function in the Fock-Darwin basis.

Since the model system used in this study is that of a harmonic confining potential, a natural choice of singleparticle basis is the set of eigenfunctions of the twodimensional harmonic oscillator in the lowest Landau level:

$$
\psi_{\ell}(z)=A_{\ell} z^{\ell} e^{-|z|^{2}} .
$$

The many-body wave function is then built in the FockDarwin basis formed from these single-particle states, and the Hamiltonian (up to an additive constant) is straightforwardly written as

$$
H=\sum_{i} \ell_{i} \hat{a}_{i}^{\dagger} \hat{a}_{i}+\frac{1}{2} \sum_{i, j, k, l} U_{i, j, k, l} \hat{a}_{i}^{\dagger} \hat{a}_{j}^{\dagger} \hat{a}_{k} \hat{a}_{l},
$$

where the interaction matrix element is

$$
\begin{aligned}
U_{i, j, k, l} & =\left\langle i j\left|\hat{U}\left(\mathbf{r}, \mathbf{r}^{\prime}\right)\right| k l\right\rangle \\
& =\iint \psi_{i}^{*}(\mathbf{r}) \psi_{j}^{*}\left(\mathbf{r}^{\prime}\right) U\left(\mathbf{r}, \mathbf{r}^{\prime}\right) \psi_{k}(\mathbf{r}) \psi_{l}\left(\mathbf{r}^{\prime}\right) d \mathbf{r} d \mathbf{r}^{\prime}
\end{aligned}
$$

Since we are working with many-body systems at given total angular momentum $L$, the one-body term yields the same energy contribution for all allowed Fock states. Therefore we may drop this term, and diagonalize only the interaction part of the Hamiltonian. Working in the lowest Landau level already guarantees that we have a finite number of

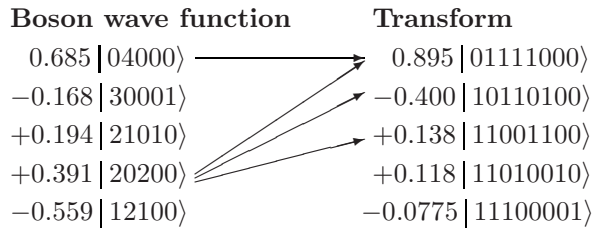

FIG. 1. As an example, we show the transformation of the $L_{B}$ $=4$ boson wave function into a fermionic wave function with $L_{F}$ $=10$. One term in the boson wave function may contribute to the weight of several terms in the transformed wave function. Correspondingly, terms in the transformed wave function may receive contributions from more than one term in the original wave function.

possible Fock states for any given total angular momentum, and in this study we have used all of these in the basis. Thus no further truncation of the Hilbert space is done. The resulting matrix is diagonalized numerically, yielding the interaction-energy spectrum and the many-body eigenstates. The diagonalization is performed using the Lanczos algorithm [38], except at the smallest angular momenta, where a full diagonalization is needed due to the small matrix sizes.

The exact-diagonalization method outlined above is applicable to both fermions and bosons; only the commutation relations of the creation and annihilation operators differ, and the many-body Fock-Darwin basis states must be given the appropriate symmetry properties (Slater determinants for fermions, product states for bosons).

The numerical implementation of the transformation, Eq. (5), requires some care. A general bosonic many-body wave function consists of a linear combination of a (possibly very large) number of Fock states. Upon multiplication by the determinant $D^{F}[(\mathrm{Eq}$. (4)], each bosonic Fock state is transformed into a fermionic many-body wave function, which is in general not a basis state in the fermion Fock-Darwin basis, but instead must be represented as a linear combination of fermionic basis states. Thus each Fock state in the boson wave function will yield several Fock states in the final fermionic wave function, and correspondingly, the weight of each Fock state in the final fermionic wave function may receive contributions from several Fock states in the original bosonic wave function.

For example, the four-particle bosonic state $|202\rangle$ (with angular momentum $L_{B}=4$ ) transforms into the fermionic wave function $0.79|110011\rangle-0.56|101101\rangle+0.25|011110\rangle$, a linear combination of three states from the Fock basis (total angular momentum $L_{F}=10$ ). The true wave function for the four-boson system with angular momentum $L_{B}=4$ is a linear combination of five basis states, as depicted in Fig. 1. The state in our toy example appears in the fourth term. Under application of the transformation, Eq. (5), this wave function transforms into the fermionic wave function shown in the right column of Fig. 1. As indicated by arrows, the bosonic state $|202\rangle$ contributes to the first three terms of the transformed wave function. Contributions come also from other terms in the original wave function. For example, contributions to the first term come from all terms of the bosonic wave function. The contribution from the boson basis state $|04000\rangle$ is also indicated in the figure (in fact, this particular 


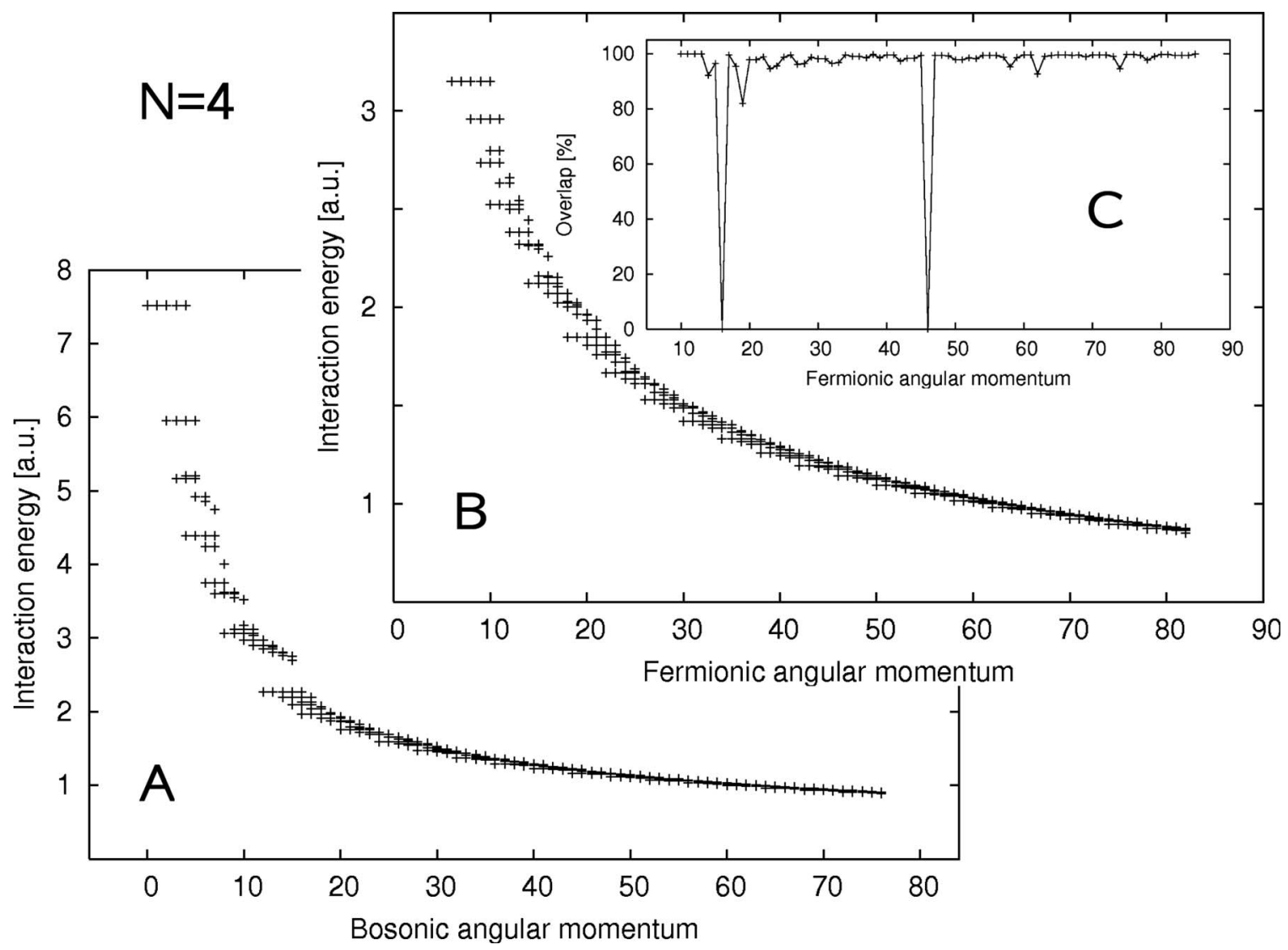

FIG. 2. Yrast spectra and overlaps for a four-particle system. (a) Yrast spectrum of four harmonically confined bosons. The five lowest states for each angular momentum are included. (b) The corresponding yrast spectrum for four fermions. (c) Overlap of the transformed boson ground state with the fermion ground state, calculated from Eq. (6).

term in the boson wave function contributes only to the first term of the transformed wave function). This example shows that it is necessary to carefully collect the weights of the basis states of the fermionic wave function resulting from the transformation.

\section{RESULTS}

We perform calculations of energies, wave functions and ground-state overlaps systematically over a wide range of angular momenta for systems containing between four and eight Coulomb-interacting particles.

Figure 2 shows the overlap of the ground state of a fourfermion system with the transformed ground state of a corresponding four-boson system, for total fermion angular momenta in the range $L_{F}=18-62$. In the small four-particle system, the overlap is very good; close to $100 \%$ for all angular momenta. The top and middle panels of the figure show the yrast spectra of the five lowest states for the boson and fermion systems, respectively. The similarity of the yrast lines is readily apparent from this figure. Both systems show a fourfold periodicity, and the kinks in the yrast line-where there is also a relatively large gap between the ground state and the first excited state-appear at corresponding angular momenta, $L_{F}=L_{B}+L_{\mathrm{MDD}}$. The overlap oscillates with the same fourfold period for high angular momenta, with the overlap having a local maximum whenever there is a kink in the yrast line.

Looking at a system with more particles, the picture becomes more complicated. Figure 3 shows the ground-state overlap in the five- and six-particle systems over the entire range of total angular momenta studied, $L_{F}=L_{\mathrm{MDD}}=10$ to $L_{F}=85$ for five particles and $L_{F}=L_{\mathrm{MDD}}=15$ to $L_{F}=89$ for six. At the smallest angular momenta, the overlap is always $100 \%$ due to the fact that the Hilbert space is so small that only one or a few wave functions are possible. It is interesting to note that also in the limit of high angular momentum, the overlap between the true fermion ground state and the transformed boson ground state tends toward 100\%. This shows that the performance of Eq. (5) is not a consequence of the Laughlin wave function being a good approximation. In fact, the overlap between the Laughlin wave function and the exact wave function decreases with decreasing filling factor [39]. Figure 3 also shows some cases where the overlap drops to zero. These cases will be discussed in some detail later in this section.

Away from the extremes of very small and very large angular momenta, the overlap drops and begins to fluctuate. In the case of five particles (top panel of Fig. 3) this is seen as a general decrease of the overlap for angular momenta in the range $L_{F}=14-35$ together with two drops to zero at $L_{F}$ $=16$ and $L_{F}=46$. This pattern is repeated in a much more complicated picture in the six-particle case, shown in Fig. 3, 

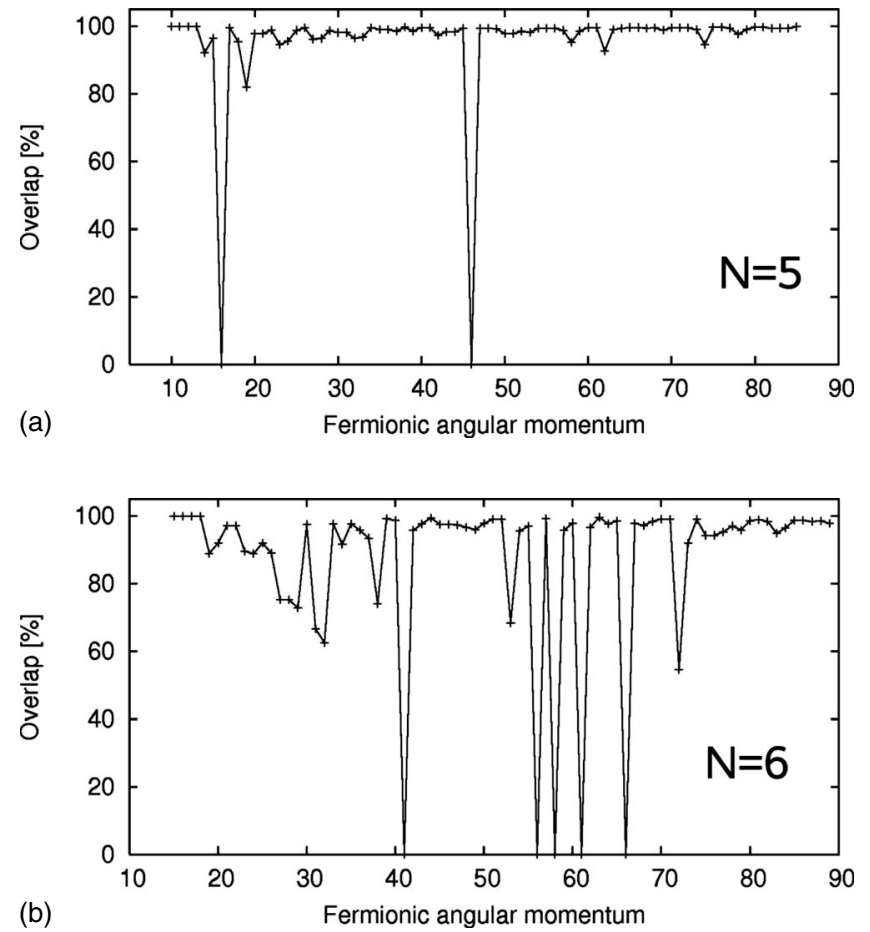

FIG. 3. Overlap between the true fermion ground state and the transformed boson ground state as a function of total angular momentum for five (top panel) and six (bottom panel) particles.

bottom panel. Again, there is a general decrease in the overlap as the total angular momentum increases beyond the small angular momenta where the overlap is large due to the small Hilbert space. As the angular momentum increases beyond $L_{F} \approx 40$ there is a region where the overlap tends to be either large or vanishing. At the very largest angular momenta studied, the overlap settles at over $90 \%$.

The behavior described for the five- and six-particle systems is echoed in smaller and larger systems as well. In the four-particle system (Fig. 2) the overlap is well above $90 \%$ for all angular momenta studied, $L_{F}=6-82$. However, for angular momenta in the range $L_{F}=10-40$ the overlap oscillates between $96 \%$ and $99 \%$, and for $L_{F}=24$ dropping to $93 \%$, before returning to values very close to $100 \%$ for larger angular momenta. In systems with seven and eight particles (Fig. 4) the overlap oscillates wildly over most of the studied region of angular momentum, where overlaps at or above $80 \%$ are interspersed with drops to about $50 \%$ or below. There is a tendency toward better overlaps in the high angular momentum limit of the data. A similar trend has been noted in composite-fermion studies of dipole-interacting fermions [34] and in a composite-fermion model of rotating Bose-Einstein condensates [36]. There is reason to suspect that the phenomena at mid angular momentum share a common origin. This speculation would, however, need to be confirmed by a detailed investigation.

We may conclude from these observations that the transformation defined in Eq. (5) from a bosonic many-body wave function to a fermionic wave function for the same number of particles and angular momentum $L_{F}=L_{B}+L_{\mathrm{MDD}}$ performs well in the low and high angular momentum limits, where good performance may be expected due to the re-
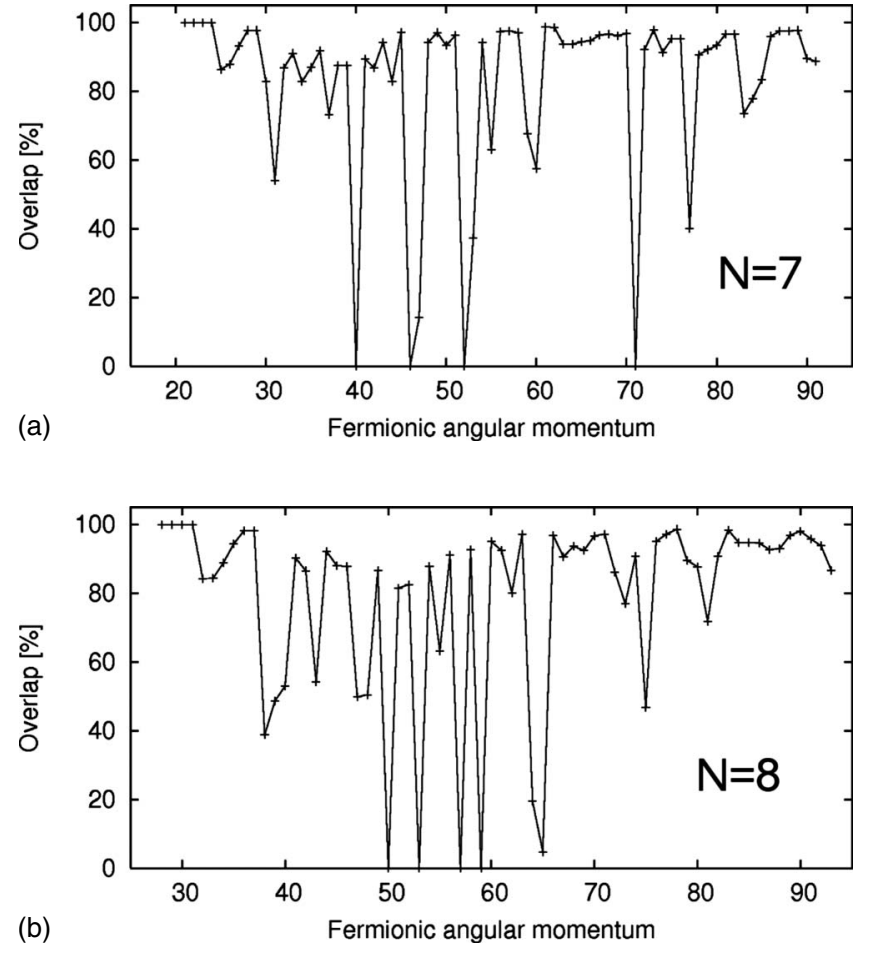

FIG. 4. Overlap between the true fermion ground state and the transformed boson ground state as a function of total angular momentum for seven (top panel) and eight (bottom panel) particles.

stricted Hilbert space and particle localization, respectively. For intermediate values of the angular momentum, the performance of the transformation needs a deeper study.

The poor performance of Eq. (5) for certain combinations of particle number and angular momentum is a result of a restructuring of levels in the yrast spectrum between the boson and fermion systems. The result is that the boson ground state will have a different structure than the fermion state, and therefore so will the transformed boson wave function. In order to study the particle configurations corresponding to the different many-body wave functions, we look at the paircorrelation function

$$
g\left(\mathbf{r}^{\prime}, \mathbf{r}\right)=\left\langle\Psi\left|\hat{\psi}^{\dagger}\left(\mathbf{r}^{\prime}\right) \hat{\psi}^{\dagger}(\mathbf{r}) \hat{\psi}\left(\mathbf{r}^{\prime}\right) \hat{\psi}(\mathbf{r})\right| \Psi\right\rangle
$$

which gives the probability of finding a particle at $\mathbf{r}$, given that there is a particle at $\mathbf{r}^{\prime}$.

We may compare the pair-correlation functions obtained from the true fermion wave function and from the transformed boson wave function. Six particles at total fermionic angular momentum $L_{F}=57$ and $L_{F}=58$ are two examples from the intermediate angular momentum range where the transformed boson ground state sometimes reproduces the fermion ground state very well, but sometimes fails spectacularly. The former is the case at $L_{F}=57$, while the latter is true for $L_{F}=58$. Figure 5 shows the pair-correlation functions of the boson wave function (left panel), the transformed boson wave function (middle panel), and the fermion wave function (right panel) for $L_{F}=57$ (top row) and $L_{F}=58$ (bottom row). The reference particle in each plot is indicated by 


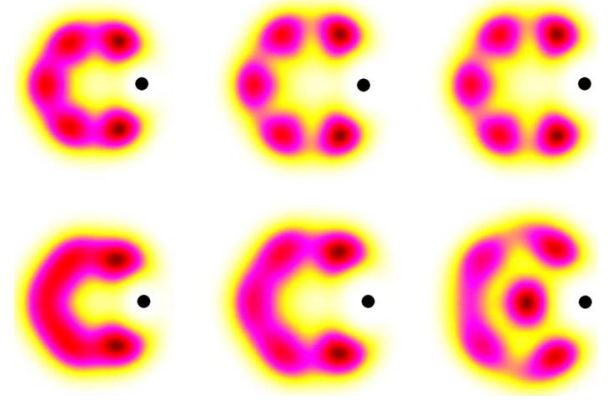

FIG. 5. (Color online) Pair-correlation functions for the sixparticle system at total angular momentum $L_{F}=57$, corresponding to $L_{B}=42$ in the boson system (top row), and at $L_{F}=58$ (bottom row). The panels in each row show, from left to right, the paircorrelation function of the boson ground state, the transformed boson ground state, and the fermion ground state, respectively. The black dot indicates the position of the fixed reference particle.

a black dot in the figure. Its position is chosen such that it sits at the distance $r_{\max }$ from the origin where the particle density has its maximum.

At $L_{F}=57$ the particle structure is extremely well reproduced by the transformation from the bosonic state with $L_{B}$ $=L_{F}-L_{\mathrm{MDD}}=42$, and the two pair-correlation functions from the true fermion wave function and the transformed boson wave function are almost indistinguishable. The true fermionic many-body wave function displays an internal structure of localized particles, where the six particles of the system are equidistantly situated on a circle. This is one of two classically (meta)stable configurations for six Coulombinteracting particles in a harmonic confining potential [40]. This structure is also seen in the bosonic wave function, and the effect of multiplication with $D^{F}$, apart from changing the particle-exchange symmetry, is to push the particles outward.

The other classically stable configuration of six equal electrical charges is a ring of five particles with the sixth particle sitting precisely at the center of the ring. In the classical system, this configuration actually has a slightly lower energy than the ring of six particles [40]. These two configurations, $(6,0)$ and $(5,1)$, are known to compete in quantummechanical six-body systems with repulsive Coulomb interaction $[41,42]$. In an electron system where the spin degree of freedom is not frozen out, the $(6,0)$ configuration tends to be favored (unless the system is very dilute), because of frustration in the $(5,1)$ configuration [41-43]. In the present study, we deal with systems of spinless bosons and of spinpolarized fermions. Therefore spin frustration does not come into play, and the two classical configurations compete. When the angular momentum is increased just by one unit to $L_{F}=58$, the fermion ground state is the $(5,1)$ configuration, as shown in the bottom right panel of Fig. 5. In the boson system, however, another change takes place with the corresponding increase of one unit of angular momentum: the particles remain seated on one ring of six particles, but the degree of localization decreases. Correspondingly, the multiplication with $D^{F}$ expands the state to a ring of six slightly smeared out maxima, a state that is orthogonal to the true ground state.
TABLE I. Overlap of the fermion ground state with the transformed wave function of each of the five lowest states of the boson system (middle column), in the system with $N=6$ particles and fermionic angular momentum $L_{F}=58$. The table also shows the overlap between the transformed boson ground state with each of the five lowest fermion states in the same system (right column).

\begin{tabular}{lcc}
\hline \hline$s$ & $\left|\left\langle\Psi_{\text {g.s. }}^{F} \mid \Psi_{s}^{B}\right\rangle\right|^{2}[\%]$ & $\left|\left\langle\Psi_{s}^{F} \mid \Psi_{\text {g.s. }}^{B}\right\rangle\right|^{2}[\%]$ \\
\hline g.s. & 0.00 & 0.00 \\
1st & 96.94 & 99.31 \\
2nd & 0.46 & 0.00 \\
3rd & 0.00 & 0.00 \\
4th & 0.00 & 0.00 \\
\hline \hline
\end{tabular}

Indeed the orthogonality of the transformed state to the true fermion ground state is no coincidence, as in fact the transformed bosonic ground state corresponds to the first excited state of the fermion system, and correspondingly the fermion ground state is well reproduced by applying the transformation to the first excited state of the boson system. Table I shows the overlap of the fermion ground-state wave function with each of the transformed wave functions of the five lowest boson states. The same table also shows the overlap of the transformed boson ground-state wave function with each of the five lowest states of the fermion system. As can be seen from the table, the transformed bosonic ground state almost perfectly reproduces the first excited state of the fermion system. Plotting the pair-correlation functions, as done in Fig. 6, confirms this picture. This figure shows the pair-correlation functions of the first excited state in the boson system and the transformed version of this state, and the first excited fermionic state. These plots may be compared with the corresponding ground-state pair correlations in Fig. 5.

$N=6, L_{F}=58$ discussed above is a particularly clean example where the many-body configurations exchange places between the fermion and the boson spectra. More generally, states may mix, such that one fermionic state is reproduced by some linear combination of transformed boson states. Such mixing of states between the bosonic and fermionic systems accounts for the cases where the overlap between the ground states is neither close to $100 \%$ nor vanishing. One example is $N=6$ particles at angular momentum $L_{F}=53$, where the overlap between the ground states is $68.32 \%$. In
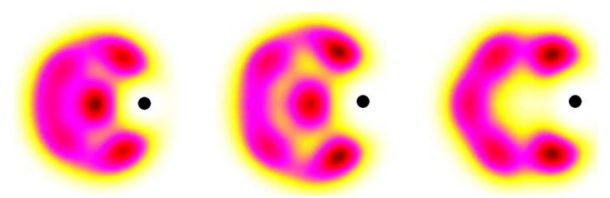

FIG. 6. (Color online) Pair-correlation functions for the first excited state in the six-particle system at total angular momentum $L_{F}=58$, corresponding to $L_{B}=43$ in the boson system. The panels show the pair-correlation function of the boson wave function (left), the transformed boson wave function (center), and the fermion wave function (right), respectively. The black dot indicates the position of the fixed reference particle. 
TABLE II. Overlap of the fermion ground state with the transformed wave function of each of the five lowest states of the boson system (middle column), in the system with $N=6$ particles and fermionic angular momentum $L_{F}=53$. The table also shows the overlap between the transformed boson ground state with each of the five lowest fermion states in the same system (right column).

\begin{tabular}{lcc}
\hline \hline$s$ & $\left|\left\langle\Psi_{\text {g.s. }}^{F} \mid \Psi_{s}^{B}\right\rangle\right|^{2}[\%]$ & $\left|\left\langle\Psi_{s}^{F} \mid \Psi_{\text {g.s. }}^{B}\right\rangle\right|^{2}[\%]$ \\
\hline g.s. & 68.32 & 68.32 \\
1st & 25.74 & 20.71 \\
2nd & 4.67 & 9.29 \\
3rd & 0.00 & 0.00 \\
4th & 0.00 & 0.00 \\
\hline \hline
\end{tabular}

this case the fermionic ground state has a considerable overlap with all of the three lowest bosonic states. This is shown in Table II. The pair-correlation functions are shown in Fig. 7. The transformed boson ground state displays a smearedout $(5,1)$ configuration, while the fermion ground state is a $(6,0)$ configuration distorted such that there is also an increased particle density at the origin.

The described mixing of the order of the states between bosonic and fermionic systems is seen also in the other cases were the overlap between the ground states is bad, also for other particle numbers than 6. There are many examples at all particle numbers larger than 4 . One particularly drastic example for eight particles is $L_{F}=57$, where the fermion ground state has $91.20 \%$ overlap with the transformed wave function of the first excited boson state. However, the transformed bosonic ground state does not have any appreciable overlap $(1.94 \%)$ with the first excited fermion state. Instead it corresponds more closely to the second excited state (with an overlap of $89.91 \%$ ).

We have seen that when the particle number increases the overlap between the fermion and boson states is still good, but in many cases the lowest energy state of the fermion system corresponds to an excited state of the boson system and vice versa. With increasing particle number the exchange of the order of levels becomes more important. This is already known in connection with the study of vortices in boson and fermion systems [17]. For example, in the boson case the single vortex reaches the origin, while in the fermion case the state with one vortex at the origin becomes an excited state when the particle number is about 10 or greater.

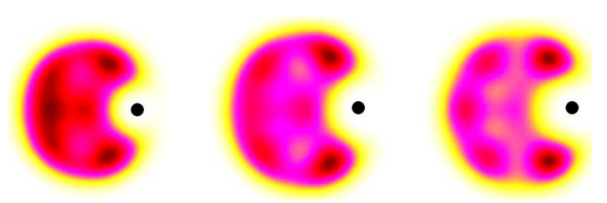

FIG. 7. (Color online) Pair-correlation functions for the sixparticle system at total angular momentum $L_{F}=53$, corresponding to $L_{B}=38$ in the boson system. The panels show the pair-correlation function of the boson ground state (left), the transformed boson ground state (center), and the fermion ground state (right), respectively. The black dot indicates the position of the fixed reference particle.

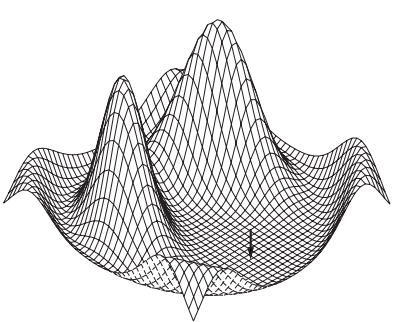

20 FERMIONS, L $=238$

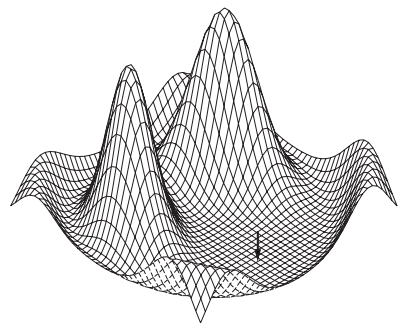

20 BOSONS, $L=48$
FIG. 8. Hole-hole pair-correlation function describing the similar vortex localization in boson and fermion systems. The boson wave function was first converted to a fermion wave function as described in the text.

This is also true for states with multiple vortices: the same vortex states are there in both systems, but they appear as the lowest state at different (relative) angular momenta. As an example, we may study three vortices in a rotating system of 20 particles. For bosons the angular momentum is 48 and the state is the lowest-energy state, while for fermions it is the fourth state at angular momentum 238. Figure 8 shows the hole-hole correlation [44] (i.e., pair correlation of vortices) which can be calculated for the fermionic wave functions [for the boson system, the transformation, Eq. (5), is first applied to the wave function]. With this large number of particles we were not able to perform the computation with the full Fock-Darwin basis. The resulting overlap between the converted boson and the fermion wave functions is only $88 \%$, but yet the resulting pair-correlation functions in Fig. 8 are nearly indistinguishable.

One may speculate that the large overlaps between fermionic wave function and transformed bosonic wave function occur when the wave function is particularly simple. One possible measure of the complexity of the fermion wave function within the given single-particle basis (here the harmonic-oscillator eigenstates) is the number of Fock states needed to make up $50 \%$ of its norm. For six particles, this number is 5 or below for angular momenta up to $L_{F}=30$, and the overlap is $70 \%$ and above, but we see very large overlaps also when the number of Fock states needed for $50 \%$ of the norm of the fermion wave function is larger than 40. This shows that the good overlaps between fermion state and transformed boson state are not merely the result of simple wave functions, but that the transformation defined by Eq. (5) can handle also complex many body states.

The results detailed this far may to some extent be robust against changes in the details of the repulsive interaction between the constituent particles of the system. This is suggested by results where we calculate the overlap between the ground-state wave function for Coulomb-interacting fermions and the transformed ground-state wave function of bosons with a repulsive $\delta$-type interaction. This is done for the case of six particles at angular momenta between $L_{F}$ $=15$ and $L_{F}=45$. The overlap is plotted as a function of $L_{F}$ in Fig. 9. This figure should be compared with the same range of angular momentum in the bottom panel of Fig. 3. For the lower half of the range of angular momenta, the overlap where the $\delta$ interaction is used for the bosons does not deviate substantially from the overlap where both systems have 


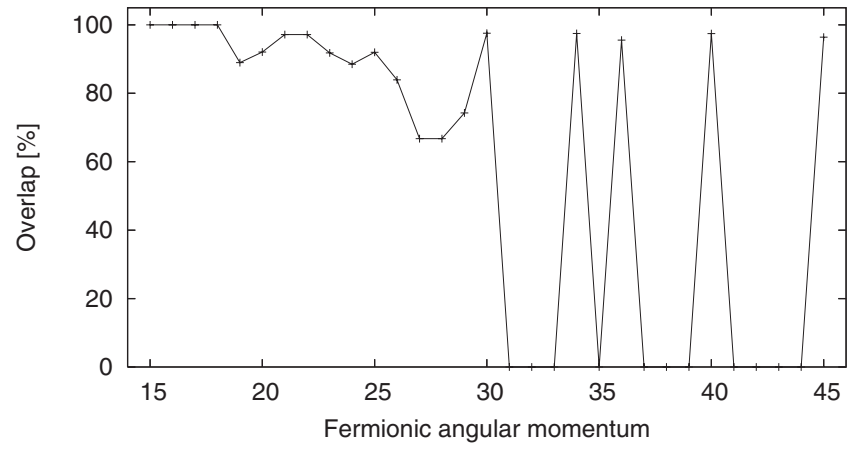

FIG. 9. Overlap between the ground state of six Coulombinteracting fermions and the transformed ground state of six bosons with $\delta$ interaction, plotted as a function of total angular momentum in the fermion system.

Coulomb interaction. For these angular momenta, the greater part of the many-body wave function comes from only a few Fock states and therefore we may expect large overlaps regardless of the type of repulsive interaction, merely due to the small Hilbert space. It is interesting to note that not only are the overlaps large (over $75 \%$ ), but the overlaps obtained from $\delta$-interacting bosons follow those obtained from Coulomb-interacting bosons closely.

These results should be expected from what we know about the Laughlin wave function. The Laughlin wave function is a good approximation to the exact many-body state as long as the angular momentum is not too large. Laughlin's construction makes no explicit reference to the details of the repulsive interaction between the constituent particles [30].

For angular momenta $L_{F} \gtrsim 30$ the overlap drops to zero except for certain angular momenta, where the overlap is very good (95\% and above), and very close to the overlap obtained when Coulomb interaction is used. In many cases the zero overlap is again simply the cause of different order- ings of the states in the two systems, but in some cases also a substantial mixing of states was found.

\section{CONCLUSION}

We conclude that for particles in the lowest Landau level there is a far-reaching universality between bosons and fermions in the properties of the rotating systems. This universality may be formulated mathematically as a transformation [Eq. (5)] from a bosonic many-body wave function to a fermionic one. These two wave functions will differ in total angular momentum exactly by $L_{\mathrm{MDD}}$, the smallest possible angular momentum in the fermion system. The transformation produces a very good correspondence (as measured by calculating the overlap integral) between the bosonic and fermionic states when the number of particles is small, when the angular momentum is very small (due to the restricted Hilbert space), and when the angular momentum is large (due to localization into states well described by Laughlin wave functions). Away from these extremes, the correspondence between boson and fermion states is more complicated. Apart from a general decrease of the overlaps due to the difficulty of reproducing the details of the more complicated wave functions as the particle number increases, there are also fluctuations between large and very small overlaps between the ground states. This is due to a reshuffling of the levels in the spectrum between the boson and fermion systems. In these cases it is not the ground state but a low-lying excited state that transforms to the fermion ground states.

\section{ACKNOWLEDGMENTS}

This work was financially supported by the Swedish Research Council, the Swedish Foundation for Strategic Research, the Academy of Finland, and NordForsk.
[1] N. K. Wilkin, J. M. F. Gunn, and R. A. Smith, Phys. Rev. Lett. 80, 2265 (1998).

[2] F. Dalfovo, S. Giorgini, L. P. Pitaevskii, and S. Stringari, Rev. Mod. Phys. 71, 463 (1999).

[3] D. A. Butts and D. S. Rokhsar, Nature (London) 397, 327 (1999).

[4] B. Mottelson, Phys. Rev. Lett. 83, 2695 (1999).

[5] G. F. Bertsch and T. Papenbrock, Phys. Rev. Lett. 83, 5412 (1999).

[6] M. R. Matthews, B. P. Anderson, P. C. Haljan, D. S. Hall, C. E. Wieman, and E. A. Cornell, Phys. Rev. Lett. 83, 2498 (1999).

[7] N. K. Wilkin and J. M. F. Gunn, Phys. Rev. Lett. 84, 6 (2000).

[8] G. M. Kavoulakis, B. Mottelson, and C. J. Pethick, Phys. Rev. A 62, 063605 (2000).

[9] I. Romanovsky, C. Yannouleas, and U. Landman, Phys. Rev. Lett. 93, 230405 (2004).

[10] M. Toreblad, M. Borgh, M. Koskinen, M. Manninen, and S. M. Reimann, Phys. Rev. Lett. 93, 090407 (2004).

[11] G. S. Jeon, C.-C. Chang, and J. K. Jain, J. Phys.: Condens.
Matter 16, L271 (2004).

[12] C.-C. Chang, G. S. Jeon, and J. K. Jain, Phys. Rev. Lett. 94, 016809 (2005).

[13] C.-C. Chang, N. Regnault, T. Jolicoeur, and J. K. Jain, Phys. Rev. A 72, 013611 (2005).

[14] M. A. Cazalilla, N. Barber'an, and N. R. Cooper, Phys. Rev. B 71, 121303(R) (2005).

[15] G. S. Jeon, A. D. Güçlü, C. J. Umrigar, and J. K. Jain, Phys. Rev. B 72, 245312 (2005).

[16] N. Barberán, M. Lewenstein, K. Osterloh, and D. Dagnino, Phys. Rev. A 73, 063623 (2006).

[17] S. M. Reimann, M. Koskinen, Y. Yu, and M. Manninen, Phys. Rev. A 74, 043603 (2006).

[18] N. Regnault, C.-C. Chang, T. Jolicoeur, and J. K. Jain, J. Phys. B 39, S89 (2006).

[19] C. Yannouleas and U. Landman, Proc. Natl. Acad. Sci. U.S.A. 103, 10600 (2006).

[20] G. S. Jeon, C.-C. Chang, and J. K. Jain, Eur. Phys. J. B 55, 271 (2007). 
[21] C. Shi, G. S. Jeon, and J. K. Jain, Phys. Rev. B 75, 165302 (2007)

[22] Z. Dai, J.-L. Zhu, N. Yang, and Y. Wang, Phys. Rev. B 76, 085308 (2007).

[23] S. Viefers, e-print arXiv:0801.4856v1.

[24] C. N. Cohen-Tannoudji, Rev. Mod. Phys. 70, 707 (1998).

[25] P. G. de Gennes, Superconductivity of Metals and Alloys (Westview Press, Boulder, 1999).

[26] A. A. Abrikosov, Sov. Phys. JETP 5, 1174 (1957).

[27] S. Bargi, G. M. Kavoulakis, and S. M. Reimann, Phys. Rev. A 73, 033613 (2006).

[28] S. Bargi, J. Christensson, G. M. Kavoulakis, and S. M. Reimann, Phys. Rev. Lett. 98, 130403 (2007).

[29] M. Koskinen, S. M. Reimann, J.-P. Nikkarila, and M. Manninen, J. Phys.: Condens. Matter 19, 076211 (2007).

[30] R. B. Laughlin, Phys. Rev. Lett. 50, 1395 (1983).

[31] S. C. Zhang, T. H. Hansson, and S. Kivelson, Phys. Rev. Lett. 62, 82 (1989).

[32] G. Murthy and R. Shankar, Rev. Mod. Phys. 75, 1101 (2003).

[33] J. K. Jain, Phys. Rev. Lett. 63, 199 (1989).

[34] K. Osterloh, N. Barberán, and M. Lewenstein, Phys. Rev. Lett. 99, 160403 (2007).
[35] A. H. MacDonald, S. R. E. Yang, and M. D. Johnson, Aust. J. Phys. 46, 345 (1993).

[36] N. R. Cooper and N. K. Wilkin, Phys. Rev. B 60, R16279 (1999).

[37] V. Ruuska and M. Manninen, Phys. Rev. B 72, 153309 (2005).

[38] R. B. Lehoucq, D. C. Sorensen, and Y. Yang, Arpack User's Guide: Solution to Large Scale Eigenvalue Problems with Implicitly Restarted Arnoldi Methods, FORTRAN Code http:// www.caam.rice.edu/software/ARPACK.

[39] C. Yannouleas and U. Landman, Phys. Rev. B 68, 035326 (2003).

[40] F. Bolton and U. Rossler, Superlattices Microstruct. 13, 139 (1993).

[41] S. M. Reimann, M. Koskinen, and M. Manninen, Phys. Rev. B 62, 8108 (2000).

[42] B. Reusch, W. Häusler, and H. Grabert, Phys. Rev. B 63, 113313 (2001).

[43] A. Ghosal, A. D. Güçlü, C. J. Umrigar, D. Ullmo, and H. U. Baranger, Phys. Rev. B 76, 085341 (2007).

[44] M. Manninen, S. M. Reimann, M. Koskinen, Y. Yu, and M. Toreblad, Phys. Rev. Lett. 94, 106405 (2005). 